\title{
Die Entgrenzung des Banalen: Zur virtuellen Beobachtung des Embodiment in der Robotik
}

\author{
Just to be simple again. . .
}

\section{Die Entgrenzung der Robotik}

Wie nur wenige andere Themen war die Robotik in den letzten zehn Jahren sowohl in den massenmedialen Berichterstattungen als auch im wissenschaftlichen Diskurs über mehrere Fachgrenzen hinweg präsent. Die real existierende oder mögliche Ankunft von robotischen Systemen in diversen gesellschaftlichen Bereichen führt zu einer parallel verlaufenden Akkumulation von Fachwissen über die Konstruktion solcher Systeme, aber vor allem über ihre Integration in die jeweiligen Organisationen. Unter dem Label einer neuen „Künstlichen Intelligenz“ $(\mathrm{KI})^{2}$ haben sich neue Disziplinen wie Machine Learning oder Data Science versammelt, in denen KI-Systeme auf Grundlage großer Datenbestände (Big Data) für potentielle gesellschaftliche Anwendungen entwickelt werden. Dabei dienen diese Datenbestände (im Fachterminus training data genannt) der Optimierung eines algorithmisch gesteuerten Verfahrens, indem sie auf eine Zielfunktion (target function) hin abgesucht, klassifiziert und für die jeweilige Situation abrufbar gemacht werden. ${ }^{3}$ In der industriellen Robo-

1 Aus dem Lied von Songs: Ohia „Just Be Simple“, 2003, Secretly Canadian Records.

2 Repräsentativ hierfür das Themenheft der Zeitschrift für Medienwissenschaft „Künstliche Intelligenzen“, besonders die Einleitung, die für eine Verbindung der gesellschaftlichen Relevanz einer neuen KI und der medialen Prägung Letzterer argumentiert (Ernst et al. 2019). Eine weitere Reaktion auf diese neue Relevanz der KI ist die Diskussion um Transparenz algorithmischer Verfahren und ein damit einhergehender, wissenschaftstheoretischer und wissenssoziologischer Diskurs um erklärbare KI („,explainable AI“, sh. dazu Miller 2019; Zerilli 2019).

3 Solche Machine Learning-Verfahren können sich von alltäglichen Anwendungen wie dem Spam-Mail-Filter bis hin zu sensorbasierten Systemen erstrecken, wie es besonders für autonome Autos der Fall ist (für den speziellen Fall der autonom fahrenden Autos sh. Weber und Kröger 2014; Stilgoe 2018; Sprenger 2019, Kap. 7.1.). Zur Anwendung des NAND-Algorithmus (Not-And) bei Spam-Mail-Filtern (sh. Mackenzie 2017, 24-25 und 113-114.). Zum sogenannten „mapping“ der Umgebung bei simultaner Lokalisierbarkeit im Raum siehe besonders die Funktionsweise so genannter SLAM-Algorithmen (Simultaneous Localization and Mapping 
tik haben verbesserte Sensortechniken dazu geführt, Roboter nicht mehr durch einen Sicherheitszaun samt Laserschranke abzugrenzen. Für physische Interaktionen mit dem Menschen erfordert dies neue Sicherheitsstandards, deren Richtwerte zunächst in Computer- und Materialsimulationen zu ermitteln sind (Haddadin et al. 2013; Kasprowicz 2018). Zugleich ermöglichten die verbesserte Sensortechnologie und die Machine Learning-Verfahren, Roboter in komplexe soziale Situationen, wie z. B. in eine Kindergartengruppe, als sozialen „Partner“ $\mathrm{zu}$ integrieren (vgl. dazu Cañameo und Lewis 2016). ${ }^{4}$ Ethische Fragen, ob es sich bei Robotern auch um eine Persönlichkeit handelt, der physisch oder psychisch Leid zugefügt werden darf, gehören seitdem zum populärwissenschaftlichen wie akademischen Diskurs dazu.

Es soll aber im Folgenden nicht um eine Ankunft des sozialen Roboters gehen, auch nicht, unter welchen Aspekten dies verifiziert werden könnte. Stattdessen wird die Mensch-Roboter-Interaktion mit Hilfe zweier Begriffe erörtert, die beide kontraintuitiv, wenn nicht gar anachronistisch auf die heutige, vom KI-Diskurs geprägte Robotik wirken: die „Banalität“ und die „Virtualität“. Was durch den Einzug von Robotern in die besagten gesellschaftlichen Bereiche notwendig wird, ist eine Banalisierung der eigenen sozialen - und das heißt vor allem - körperlichen Interaktionen und deren Erwartungen. Es wird argumentiert, dass mit dieser Banalisierung mehr als ein Reduktionismus von Bewegungen auf Kräfte und Sensoren einhergeht, sondern die Einführung einer Beobachtungsebene, einer virtuellen Beobachtung, die durch Medien der Virtualität (wie der Virtual Reality, aber auch Computersimulationen oder materielle Testreihen) gekennzeichnet ist. Sicherlich sind virtuelle Beobachtungen häufig Testpraktiken im Konstruktionsentwurf (Thiele et al. 2020; Schirra 2009), aber sie sind mit Blick auf die Mensch-Roboter-Interaktion auch der Ort, an dem die Unwahrscheinlichkeit einer Kommunikation kognitiver und nicht-kognitiver Agenten wahrscheinlich gehalten werden soll - oder, um es mit Elena Esposito zu sagen, die es allgemein für algorithmisch gesteuerte Maschinen so formulierte: „The paradoxical purpose of programming intelligent algorithms is to build unpredictable machines in a controlled way. The goal is to control the lack of control" (Esposito 2017, 257). Banalität konzentriert damit eine Reduktion von Komplexitäten interagierender Systeme, die für die Wahrscheinlichkeit der Interaktion notwendig ist und die erst im Medium der Virtualität beobachtbar wird.

Technology), die sowohl für Fahrzeuge als auch für mobile Roboter zunehmend Verwendung finden (Kanderske und Thielmann 2019).

4 Dies geht oft mit einer multidisziplinären Ausrichtung im Roboter-Design einher, die neben Ingenieur`innen auch Psycholog^innen und Expertisen aus neuen Feldern wie den developmental robotics einbeziehen. 
Es geht folglich nicht darum, die Mensch-Roboter-Interaktion auf einfachen, alltäglichen Bewegungen zu begründen, sondern um die Einführung der Banalität als einer Alternative zur dominierenden Kategorie der Intelligenz. ${ }^{5}$ Im Gegensatz zur Intelligenz, die sich primär auf kognitive Akte richtet und häufig anthropozentrisch verbleibt, reklamiert das Banale eine Aushandlungszone körperlicher Praktiken, die nur entstehen kann, wenn beide interagierenden Systeme den Anderen für ihre Operationen einbeziehen. Damit gewinnen - im Anschluss an Niklas Luhmanns Begriff der Interpenetration - technische wie psychische Systeme als Interagierende sukzessiv an Komplexität (Luhmann 1987, 299).

Aber wie lässt sich Banalität an solche technischen Begegnungen von Mensch und Roboter rückbinden? Das Banale ist zunächst eng verbunden mit den jeweiligen Vorstellungen und Erwartungen darüber, was bekannt, einfach, vertraut und alltäglich ist. Besonders technik- und medienspezifische Erwartungen sind in jeder Nutzerkonstellation gegeben, die zugleich die Gestaltung der jeweiligen Objekte und ihre Bedienbarkeit prägen (Oudshoorn und Pinch 2005). Für den Fall von Mensch-Roboter-Interaktionen zeichnet sich aber eine zweiseitige Konstruktionslogik heraus: Denn auf der einen Seite gilt es, körperliche Interaktionen mit Robotern auf einfache, diskretisierbare Bewegungen und minimale, sozial kodierte Gesten zu reduzieren, damit sie modelliert und später im Computer simuliert werden können. Wie in allen Bewegungsstudien repräsentiert dies den Analyseteil, der notwendig für die folgende, synthetische Konstruktion jeder möglichen Bewegung ist (Kasprowicz 2019a, Kap. 4.4.1.). Auf der anderen Seite zieht gerade die Inklusion komplexer Technologien in alltägliche Situationen eine Kontingenzsteuerung nach sich (oder, Esposito paraphrasierend, einen kontrollierbaren Kontrollmangel). Damit führen neue Handlungsoptionen (und Bewegungen) mit Robotern zu neuen Wissenskategorien der körperlichen Begegnung, damit Interaktionen von Menschen und Robotern für Konstrukteur*in und Nutzer^innen wahrscheinlich oder weniger abstrakt formuliert - tolerierbar bleiben. Solche Kategorien umfassen Skalierungsbegriffe wie die Unaufdringlichkeit (Kasprowicz, in Vorb.). Sie äußern

5 Auch hierin folge ich Elena Esposito insofern, als dass sie in ihrem Modell die Kommunikation an die Stelle der Intelligenz zur entscheidenden Kategorie erhebt. Ob Maschinen mit uns kommunizieren oder ob - wie oft gefragt wird - sie uns verstehen, unterliegt somit dem Kommunikationsmodell nach Luhmann, das von der doppelten Kontingenz zweier Systeme abhängt, die den Anderen als Fremdreferenz in ihre operative Schließung inkludieren. Dies setzt bei Luhmann so genannte autologische Systeme voraus, also solche, die an ihre Unterscheidungen anschließen können und somit den Anderen als abwesenden Anwesenden in den eigenen Operationen mitführen (sh. dazu hier Punkt 5 sowie Esposito 2017, 255-257; Luhmann 1987, 367-371 und 1997a, 124-127). 
sich in einer Episteme der Technikakzeptanz, die mit den neuen Handlungsoptionen (samt deren Kontingenzen) geschaffen wird, bevor Technikakzeptanz ein quantitativ bestimmbares Phänomen werden kann. ${ }^{6}$ Unaufdringlichkeit setzt ein Wissen um die Banalität sozial kodierter Gesten und Bewegungen voraus, das sich zugleich im Spannungsfeld sozialer Erwartungen bewegt - und zwar in dem des interagierenden Menschen und jenem einer die Interaktion rahmenden Organisation wie z. B. der Fabrik, der Pflegeanstalt oder dem vom Straßenamt beaufsichtigten Verkehrswesen (zur spezifischen Rahmung der Interaktionsmöglichkeiten durch die Organisation sh. Baecker 2005, 114-117). Damit einher geht eben keine Ausblendung des Mediums. Vielmehr drängt die Unaufdringlichkeit - als Diskretionserwartung bestehender sozialer Interaktionen - das Paradoxon einer neuen Banalität auf, dass sowohl den ingenieurwissenschaftlichen Modellen als auch den Bewegungen der menschlichen Interaktionspartner zugrunde liegt. Beides setzt allerdings Medien der Virtualität voraus, in denen aus dem alltäglichen Bewegungsablauf das Banale erst beobachtbar wird.

Da gerade die Frage der Banalität eine eigene Vorgeschichte hat, die sich nicht zuletzt auch in häufig synonym verwandten Begriffen wie der Trivialität äußert, soll zunächst kurz eine begriffshistorische Herleitung des Banalen für das zwanzigste Jahrhundert erbracht werden. Anschließend werden die Phänomenologie und der von Edmund Husserls geäußerte Leitspruch „,Wir wollen auf die ,Sachen selbst“ zurückgehen“ (2009 [1901], 10) als ein ideenhistorischer Zugriff auf das Einfache als Grundlage des Erkenntnisprozesses betrachtet. Von dieser kurzen Ideengeschichte des Banalen geht es zur Systemtheorie, in der Banalität nur unter Bedingungen der doppelten Kontingenz erscheint. Damit wird eine theoretische Alternative auf die bewusstseinszentrierte Analyse des Banalen in der Phänomenologie geboten. Im letzten Teil dient eine kurze Analyse dazu, die Konstruktionslogik des Banalen in der Robotik und seine Rückbindung an eine daraus entstehende Episteme des Embodiment $\mathrm{zu}$ verdeutlichen. Es wird $\mathrm{zu}$ zeigen sein, wie die Virtualität hier zum Medium einer paradoxen Figur der komplexen Banalisierung wird, um Kommunikation zwischen kognitiven und nicht-kognitiven Agenten probabel zu gestalten.

6 Siehe zur Methodik einer Technikfolgeabschätzung für die Mensch-Roboter-Interaktion Meister 2011; Decker 2011 und ausführlich zu technischen und rechtlichen Aspekten Christaller et al. 2001. 


\section{Der Begriff des Banalen}

Auf den ersten Blick scheint das Konzept des Banalen vertraut und nicht erläuterungsbedürftig: Es manifestiert sich im Alltagsgebrauch, sei es als materielles Objekt oder als voraussetzbares Knowhow. In dieser Bedeutung folgt es seiner etymologischen Herkunft aus dem Altfranzösischen, dem „ban“, dem Gemeinwesen sowie -wissen zugehörig. Damit wurden Dienste oder Gegenstände bezeichnet, die der Lehnsherr seinem Vasallen für eine Gegenleistung überließ. Sie waren so gesehen für alle erwerblich, allerdings nur bei dem einen Lehnsherren oder Gewerbetreibenden (daher auch die Verbindung mit dem Bannrecht, also die Verbannung aus dem Gemeinwesen bei etwaiger Verletzung des Lehnsherrenrechts) (o. A. 1857, 264). Begriffe wie das „banlieu“ bezeichnen noch eine geographische Markierung, bis wohin das Bannrecht sich für das Gemeinwesen erstreckt - bis wohin also das gewöhnliche Recht des Fürsten oder Lehnsherren herrscht. Erst im neunzehnten Jahrhundert werden die Bannrechte durch die Industrialisierung und Urbanisierung aufgehoben. Aber bereits im achtzehnten Jahrhundert löst sich die Bedeutung vom „Bann“ hin zu etwas, das richtig oder geltend ist, aber so selbstverständlich, dass es nicht explizit gemacht werden muss (Genz 2011, 12). Erst im späten neunzehnten und frühen zwanzigsten Jahrhundert erhält das Banale seine negative Konnotation, die eng mit dem Aufkommen der Tagespresse und dem zusammenhängt, was heute unter Nomina wie Massenmedien und Populärkultur firmiert. So koppelt Julia Genz in ihrer Studie zu Banalität, Trivialität und Kitsch in der Literatur die Banalität nicht an die Qualität des Inhaltes, sondern an den Exklusivitätswert des literarischen Produktes und seiner soziokulturellen Zugänglichkeit. Das Banale unterliege dabei einer massenmedialen Verbreitung, während die kognitive Ebene durch den Begriff der Trivialität und die emotionale mit dem Kitsch belegt wären (Genz 2011, 16).

Das pejorative Element des Gewöhnlichen und für alle Bekannten verbleibt aber nicht allein im Distinktionshabitus von elaborierten und popkulturellen Werken und ihren Produzenten (Autor^innen, Komponist*innen, Filmen und ihren Regisseur ${ }^{\star}$ innen etc.). Es taucht auch in der Unterscheidung einer Wissensproduktion und -verarbeitung auf, die zum trennenden Merkmal zwischen einer wissenschaftlichen und einer phänomenologischen, literarisch-essayistischen Sicht auf die Welt wird. So unterscheidet Hans Blumenberg 1974 in seinen „Vorbemerkungen zum Wirklichkeitsbegriff“ den Alltag als die „Konsistenz des Gewöhnlichen“, der dem Theoretiker als das „Ungeheuere erscheinen“ muss, da er gerade nichts Neues biete (Blumenberg 1974, 10), das ein Explanandum wert wäre. Vor diesem Hintergrund sind die Helden literarisierter Banalität Autoren wie Franz Kafka, die das gesamte eruptive Potential der Moderne in einer Absur- 
dität verpacken, die immer wieder im grauen Alltag auflauert. Bis in die aktuellen Ausläufer solcher Einschätzungen hält sich diese Bedeutung des Banalen, wenn sie nicht pejorativ, sondern als Abgrenzung zu einem progressiv gewandten Wissensbegriff genutzt wird.

Dem Banalen kann man sich daher nicht ohne Vorbehalt mit dem Werkzeug von Theorien und Methoden nähern. Vertreter der Neuen Phänomenologie wie Jürgen Hasse gehen gar soweit, von einem Vakuum zu sprechen, in das alle Theorien über banale Alltagserlebnisse fallen, „[. . .] denn sie werden, indem ihnen jeder apriorische Dignitätsanspruch genommen wird, selbst zu etwas Banalem“ (Hasse 2017, 49). Das Abrutschen so hoher geistiger Leistungen wie der Theorie hin zu Phänomenen, hinter denen sich schlicht und einfach nichts verberge, verlangt nach anderen Zugängen. Für die folgende Fragestellung nach der Banalität, die im Modus der Virtualität in die Robotik eingeführt wird, ist diese Sachlage von Relevanz: Man kann sich, wie die Neue Phänomenologie, in quasi-literarische, künstlerisch-explorative Welten einer Innenschau begeben. Dieses erzählt allerdings eher von der Begegnung mit dem Banalen als von der Banalität im operativen Modus des Virtuellen. ${ }^{7}$ Will man dies vermeiden, so muss die Frage gestellt werden, was es voraussetzt, Banalität als das Einfache, als das Zugrundliegende $\mathrm{zu}$ beschreiben, ohne in die Untiefen einer subjektiven Empfindungswelt (oder ihrer Beobachtung erster Ordnung) zu rutschen. Erst vor diesem Hintergrund können Medien ins Verhältnis mit der Banalität gesetzt werden. Daher gilt es nun, zunächst von der anderen Seite zu starten. Dazu wird mit der Phänomenologie ein theoretischer Zugang präsentiert, der das Gegebene des Bewusstseins selbst zum Ausgang allen möglichen Wissens befragt. Erst im Anschluss kann die Systemtheorie Luhmanns plausibel als Entkopplung des Banalen von seiner subjektiven Fundierung begründet werden.

\section{Die Entgrenzung des Banalen}

Es wäre falsch, Husserls Anspruch, zu den Sachen selbst zurückzukehren, als Aufruf zu einer philosophischen Auseinandersetzung mit der Banalität zu interpretieren. Nichts liegt Husserl ferner als eine Banalisierung der philosophischen Praxis. Es ist eher ein langausholendes Suchen nach den Grundlagen der Erkenntnis, die noch vor jeder wissenschaftlichen Untersuchung einer „Wesensanalyse“ bedarf (Husserl 1973 [1907], 38). Da nun wissenschaftliches

7 So sind es bei Hasse vor allem Warteräume oder Lounges, die Orte eines banalen Zeiterlebens sind. 
Wissen sich bereits aus einer vorgestellten Position zu seinen Objekten und einer symbolischen Ordnung konstituiert und damit „transzendentes“ Wissen ist (Husserl 1973 [1907], 5), erfordere dies eine Reduktion, eine Freilegung auf die allgemeine Bezugnahme des Bewusstseins zu seinen Phänomenen (Intentionalität). Nur von dort aus kann der Akt der Erkenntnis erst seinen Ausgang nehmen. Die von Husserl eingeführte phänomenologische Reduktion führt dabei die Suche nach dem einfachen Ausgang aller Erkenntnis ein - dem Sein setzenden Bewusstsein. Aber auch hier ist der skeptische Phänomenologe nicht über alle Zweifel erhaben, denn: „Der Anfang war die Evidenz der cogitatio. Da schien es zunächst, als hätten wir einen festen Boden, lauter pures Sein. Man hätte einfach nur zuzugreifen und zu schauen." (Husserl 1973 [1907], 70-71).

Was jedoch hinter diesem vermeintlichen Schleier einer Gegebenheit für das Bewusstsein liegt, wird zum eigentlichen Problem und Kern der Husserlschen Phänomenologie nach 1907 - und zwar die zeitliche Verfassung des Bewusstseins selbst. Wahrnehmung und Phantasie als Bewusstseinsakte stehen selbst nicht isoliert da, sie sind eingewoben in die Erinnerungen (Retentionen) und Assoziationen der operierenden cogitatio, die zugleich auch Erwartungen für die kommenden Wahrnehmungen stiftet (Protention). Der intentionale Akt hat stets einen Gegenstand, auf den er gerichtet ist, und dieser Gegenstand ist allein unserem Bewusstsein zugänglich. Der von Husserl als Noema bezeichnete Gegenstand, oder genauer der Anhaltspunkt des Denkens, muss kein real existierendes Objekt sein (Føllesdal 1969, 681). Es ist eine Art temporale Datei, auf die neue Informationen sowie wiederkehrende Bewusstseinsakte (Wahrnehmen, Erinnern, Phantasieren) zugreifen. ${ }^{8}$ Die phänomenologische Reduktion führt auf eine einfache, allen Bewusstseinsoperationen zugrundeliegende Temporalität, die sich in unterschiedlichen Bewusstseinsakten (Wahrnehmen, Erinnern etc.) unterschiedlichen oder gleichen Gegenständen - also Noema - widmen können. Sie offenbart einen Exzess an Komplexität im Bewusstsein selbst und damit zugleich in den vermeintlich banalsten, alltäglichen Vorgängen.

8 Das Noema ist dem Bewusstsein, aber nicht durch die Sinne gegeben. Folglich kann das Noema auf real existierende Objekte verweisen, aber es ändert sich als Baum je nach dem, ob es erinnert, durch Phantasie manipuliert oder schlicht mit anderen Bäumen verglichen wurde. Auf der anderen Seite kann sich das real existierende Objekt (das Auto meines Freundes) verändern, ja verschwinden, während ich eine konsistente Vorstellung von dem einen speziellen Moment seiner Wahrnehmung habe, die ich mir immer wieder vergegenwärtige, weil ich ihr eine Bedeutung attestiere (vgl. Føllesdal 1969, 684; zum Noema als Verhältnis von Bild und Imagination sh. Sartre 1971 [1940]). 
Gerade auf solchen sich anbahnenden, von Selbst- und Fremdbezügen im Denken, von den Akten (Noesis) und ihren sinnhaften Inhalten (Noema) versehenen Vorgängen untersucht Niklas Luhmann in seinen späten Jahren Husserls Programm. Die Systemtheorie, die bei ihrer Distanz zum Subjektbegriff und zu den Untiefen des Bewusstseins nicht viel mit der Phänomenologie zu teilen vermag, setzt beim Problem Husserls an, gerade an jener Banalität des Bewusstseins von sich, diesem „pure[n] Sein“ (Husserl 1973 [1907], 70), das sich nun selbst der Kontingenz kognitiver Operationen ausgesetzt sieht (Luhmann 1997b, 34). Mehr noch - der Systemtheorie nach ist es gerade das Inkludieren einer nicht eigen-kognitiven Zeit in die Bewusstseinszeit, ebenso der Wahrnehmungen in den Bewusstseinsstrom, das eine Fremdreferenz ausmacht - damit also die zentrale Unterscheidung zwischen Selbst und Umwelt, Ich und nicht-Ich als Form der Differenz zur Erhaltung von Identität ausführt. Diese Identität (die das nicht-Identische als ihre Negation mitführt) ist daher nicht als Subjektivität, sondern als Leistung eines psychischen, sozialen oder technischen Systems zu verstehen, das Unterscheidungen trifft, in dem es selektiv beobachtet, weil nicht alles gleichzeitig beobachtet werden kann (Luhmann 2002, 227). Die Form als Einheit einer Differenz (Ich/nicht-Ich) referiert nicht mehr auf ein metaphysisches Substrat oder eine biologische Entität, sondern allein auf einen anschlussfähigen „Einschluss des Ausgeschlossenen als Modus des Prozessierens von Sinn“ (Luhmann 1997b, 56).

Kontingenz ist in der Systemtheorie allerdings nicht nur in der Reproduktion solcher Identitäten grundlegend, sondern auch im Kommunizieren von Systemen untereinander. Denn es gibt keine Einsicht in die Bewusstseinsakte des Anderen und man weiß nie, was als nächstes kommt - folglich wird Kommunikation selbst $\mathrm{zu}$ einem unwahrscheinlichen Akt. Genau diesen Umstand bezeichnet Luhmann als „doppelte Kontingenz“ (Luhmann 1987, 153-154). Um diese Kontingenz zu reduzieren, treten Medien auf den Plan, besonders „symbolisch generalisierte Kommunikationsmedien“ wie Buchstaben, die in Form von Begriffen und Sätzen zusätzliche Unterscheidungen bei gleichzeitiger Kontingenzreduktion ermöglichen, da das vergangene Wort nun schriftlich fixiert und distribuiert werden kann (Luhmann 1997a, 204). Es wäre aber ein Kurzschluss, den Medienbegriff hier allein auf Symbole zu beschränken, denn gerade in sozialen Institutionen sind Körperhaltungen und Gesten ebenso Formen einer Kommunikation. ${ }^{9}$ Mehr noch, sie sichern durch Handlungsroutinen

9 Dabei sind die Haltungen, Redetakte und Gebote des Kommens und Gehens selbst Formen der Kontingenzreduktion psychischer Systeme, die alle auf einen sozialen Code referieren, in dem die Anwesenheit und Abwesenheit in der Kommunikation (bei physischer Präsenz im selben Raum) reguliert ist (Baecker 2005, 110-111). 
die Reproduzierbarkeit von Organisationen. Damit werden aber soziale Systeme selbst zu dem, was der Kybernetiker Heinz von Foerster mit Blick auf die Schule etwas verächtlich als „Trivialisierungsmaschine“ bezeichnete (von Foerster 1997, 41), als den Ort einer systematischen Reduktion von Komplexität. ${ }^{10}$ Das Triviale, dieses getreue Synonym des Banalen, hat bei von Foerster die Grundeigenschaft des analytisch Bestimm- und damit Voraussagbaren.

Analog dazu verwendet von Foerster die Unterscheidung triviale und nichttriviale Maschine (von Foerster 1984). Die triviale Maschine vollzieht eine simple Input-Output-Logik, wie es auch noch jede`r PC- oder Smartphone-Nutzer^in heute erwartet, vorausgesetzt, die Maschine ändert in dieser Zeit nicht ihren Zustand. Nicht-Triviale Maschinen schließen in ihren Handlungen an gemachte Unterscheidungen an, sie entwickeln daraufhin einen „Eigenwert“ des Wirklichen, der mit dem Ausgangswert (dem Input) nicht mehr identisch ist (von Foerster 1984, 154). Dieser Faktor des kontingenten Zustandes eines Agenten hat aber ontologische Konsequenzen mit Blick auf die Robotik - denn nicht nur eine überraschende Bewegung des Roboters lässt ihn uns als nicht-triviale Maschine beobachten. Auch eine Dys- und Fehlfunktion überrascht uns und führt die grundlegende Unwahrscheinlichkeit von Kommunikation vor Augen. Aber im Falle der Robotik hat ein solches Scheitern der Trivialisierung bzw. Banalisierung den Effekt, den Roboter zu negieren und ihn nicht mehr als Kommunikationspartner, sondern als technisches Objekt zu händeln. Aus ethnographischer Sicht formuliert es die STS-Forscherin Morana Alač wie folgt:

I turn to robots as physical things in their own rights and suggest that for a robot to be dealt with in social situations its being perceived as not only alive but also as a material, object-like piece of the world matters. This, in turn, means that the agency of the robot is entangled with its thing-like materiality [. . .] One advantage that this approach provides is the possibility of accounting for the robot's character as multifaceted. As we move away from the idea that the robot's sociality has to be understood as an intrinsic and categorical property of the robot's inside [. . .], the robot is a technology that can be enacted, in one breath, as an agent and a thing. Each of the facets that hand-in-hand maintain each other can become the "theme“ [. . .] at specific moments in interaction while its other profile coexists concurrently or as a possibility that can itself take center stage as the encounter develops.

(Alač 2016, 520)

Solche Ansätze wie jener von Alač nehmen einfache Interaktionen mit Robotern als Ausgang für die Analyse einer multiplen Ontologie, einer Koexistenz von technischem Ding und sozialem Partner, deren Zuschreibungen selbst fragil sind (sowohl von den Interagierenden mit dem Roboter als auch von Alač selbst als

10 Für diesen Hinweis zum Begriff der Trivialisierung bei Heinz von Foerster danke ich Dirk Baecker. 
Beobachterin erster Ordnung). Empirische Studien dagegen suchen nach Parametern, anhand derer sich das Engagement für eine kollaborative Arbeit erfassen lässt, obwohl oder gerade weil es sich vor dem Versuch weiterhin um eine Maschine handelt (hier besonders für die soziale Robotik Paauwe et al. 2015). Geht man aber nicht von solchen Beobachtungen erster Ordnung aus und versucht, die Modi einer Unterscheidung Objekt/Partner selbst in den Beobachtungen der Ingenieur*innen $\mathrm{zu}$ greifen, stellt sich zwangsläufig die Frage, wie der Mensch selbst als potentieller Partner in den Roboter kommt? Gerade um eine Antwort auf solche Fragen zu finden, gilt es, Medien für eine virtuelle Beobachtung zur Verfügung zu stellen. Wie der Mensch vom Roboter „gesehen“ werden soll, wird damit eine Frage der Operationalisierung von Banalität in Medien der Virtualität. ${ }^{11}$ Anstelle der Frage, unter welchen Bedingungen Menschen die Interaktion mit Robotern aufrechterhalten oder sich darin engagiert fühlen, werden Ingenieur*innen als Beobachter von Maschinen beobachtet, die den Menschen als Fremdreferenz ihres Systems inkludieren müssen. ${ }^{12}$

\section{Die virtuelle Beobachtung der Banalität „Mensch“}

Husserls Noema wurde als zeitlicher Gegenstand des Bewusstseins benannt. Die Noema kann Referenzen zu einer Außenwelt haben, aber sie ist ein immanenter Bezugspunkt des Bewusstseins selbst. Mit der Reduktion auf die Sachen

11 Medien der Virtualität umfassen hier auch Modelle in Flow-Charts, Computersimulationen von möglichen Bewegungsabläufen sowie die besagten VR-Simulationen zur Ermittlung einer Interaktionswahrscheinlichkeit. Auf die jeweiligen Verfahren der Robotik wird jeweils konkret verwiesen, während der Modus der Virtualität zur allgemeinen Verfahrenstechnik für das Embodiment in der Robotik gehört.

12 Diese Situation ist aus epistemologischer Sicht nicht neu. Der entscheidende Aspekt ist aber das Embodiment als Konstrukt des Anderen für ein psychisches oder technisches System. Gerade die Human-Computer-Interaction (HCI) hat seit ihrer Gründung, und auch in ihrer allmählichen Abwendung von einer kognitionswissenschaftlichen Zentrierung, immer den Modus eines virtuellen Anderen als Fremdreferenz für das technische System beobachtet. Das Interface-Design ist damit auch ein Artefakt der Grundunterscheidung User/Nicht-User, aus dem die HCI ihr Wissen generiert, um die Kommunikation mit technischen Systemen wahrscheinlicher zu gestalten (sh. zu einer Diskussion zwischen Computeravataren für die HCI und Avataren für die Mensch-Roboter-Interaktion Gratch et al. 2015; zum Verständnis der HCI Bannon 2011). 
selbst drängte sich aber die Komplexität der diversen, stets temporalen und die Tempi setzenden Bewusstseinsoperationen erst auf (Bergmann und Hoffmann 1989, 156). Die Systemtheorie lieferte dabei eine Perspektive, in der nicht mehr vom Sein setzenden Bewusstsein ausgegangen wird, sondern von der Einheit der Differenz, die Form genannt wird, und deren Priorität es ist, die Stabilität des (psychischen) Systems durch Reproduktionen vertrauter und Integration neuer Differenzen zu gewährleisten. Banalisierung - oder mit Heinz von Foerster gesprochen, Trivialisierung - erwies sich hierin als ein Verfahren zur Reduktion von Komplexität. Solche Trivialisierungen, die sowohl zwischen technischen und psychischen als auch zwischen psychischen und sozialen Systemen geschehen können, setzen Beobachtungen voraus, d. h. Unterscheidungen, die auf Medien basieren. Es wurde bereits betont, dass der Medienbegriff der Systemtheorie hier nicht allein auf klassische Kommunikationsmedien, sondern auch auf Bild- und Simulationsmedien ausgeweitet wird. Vor diesem Hintergrund wäre zu fragen, wie in der Robotik eine Bezugsgröße „Mensch“ durch virtuelle Medien konstituiert wird? Und welche Rückwirkung haben diese virtuellen Medien auf das Embodiment (materialisierter) Roboter, die mit ihren menschlichen Kollaborateur^innen kommunizieren und damit ihre Sozietät beobachtbar machen sollen?

Ein Blick in die fachwissenschaftlichen Diskussionen und Methoden, aber auch in die Praktiken zur Modellierung von Menschen zeigt, dass es hier weder eine Priorität in den Verfahrenstechniken noch in den disziplinären Wissensbezügen gibt. So stehen Motion-Capture-Techniken neben 3D Bodyscans, Digitale Menschmodelle neben Machine-Learning-Verfahren und den diversen Computersimulationen von Körperbewegungen (Bortot et al. 2010; Chaumette und Hutchinson 2009; Bubb und Fritzsche 2009). Besonders VR-Simulationen für kollaborative Praktiken werden in der industriellen Robotik angewandt, wenn es um die Einschätzung adäquater Abstände und das Einüben routinierter Bewegungen geht. Diese Verfahren halten ebenso Einzug in die Sicherheitsrichtlinien der International Organization for Standardization (ISO), in denen die Kriterien für eine Unterscheidung von Service- und Industrieroboter sowie deren Leistungsbereiche vereinheitlicht werden (Onnasch et al. 2016; Gammieri et al. 2017). ${ }^{13}$

13 So ist die physische Interaktion zwischen Roboter und Mensch seit 2006 in der ISO 10218 vorgeschrieben. Sowohl für industrielle Anwendungen als auch im Dienstleistungssektor müssen dabei Leistungsgrenzen des Roboters quantitativ ermittelt werden, um jederzeit das Gefährdungspotential in einer Interaktion bestimmen zu können. Dies führt zum Teil auch dazu, dass Ingenieur*innen in den Normen Einschränkungen für die Leistungsfähigkeit und Entwicklung der Roboter durch zu restriktive Sicherheitsvorgaben sehen. Zur Diskussion um Sicherheitsnormen als Bewegungsrestriktionen für Roboter vgl. Haddadin et al. 2009. 
Aber es sind nicht nur Sicherheits- und Funktionsfragen, die in den Medien der Virtualität ihren Einzug finden. Gerade das Design einer Kontaktstelle von menschlicher und maschineller Hand sowie die dazugehörige Körperbalance sind dabei von besonderer Relevanz. Da mit dem Aufzeichnen von Gehbewegungen in der VR oft keine adäquaten Daten einhergehen, weil das Durchschreiten virtueller Welten ungewöhnlich für Probanden ist und den Gang ins Stocken bringt, wird nur die obere Körperpartie in Interaktion mit einem Greifarmroboter getrackt (Thomasset et al. 2019). Dabei dienen die haptischen Kontakte und Bewegungen des Menschen als Datenmaterial für die Bestimmung eines kinästhetischen Verlaufs. Solche Verfahren einer kinästhetischen Simulation einfacher Übergabebewegungen oder der kooperativen Arbeit mehrerer Hände an einem Tisch finden auch in sogenannten „virtual tool dynamics“ Anwendung (Tykal et al. 2018). Sie sollen die Banalität quasi auf Demonstrationsebene bringen, in dem der Bewegungsverlauf nicht in die Maschine per Programmcode einschrieben, sondern allein aus der Führung durch den User, der das „kinaesthetic teaching“ anleitet, generiert wird (Tykal et al. 2016, 205).

Neben solchen VR-Testumgebungen gibt es zunehmend Machine LearningVerfahren, die auf den gesamten Körper ausgerichtet sind (vgl. Tekin et al. 2016). Dabei sammeln die Ingenieur^innen Trainingsdaten von menschlichen Körperposen aus verschiedenen Perspektiven, zum Teil mit wechselnden Lichtverhältnissen. Solche training data können sowohl per Bodyscanner entstehen oder per Motion-Capture-Verfahren, aber es ist genauso möglich, einen großen Bestand an digitalisierten Photographien hierfür zu verwenden. Mittels solcher Datenbänke lassen sich neuronale Netzwerke trainieren, anhand derer auch schnell ausgeführte Wechsel von Körperposen oder Überlagerungen von Körperteilen - so genannte Occlusions (Sárándi et al. 2018) - rechtzeitig erkannt werden sollen. Noch bevor das „Training“ mit den Datenbeständen geschieht, müssen beim Programmieren die Parameter für die Körperposen festgelegt werden, d.h. Grenzen, bis wohin es sich um ein Knie oder einen Kopf halten würde, und welche Pose es wäre, wenn ein Kopf z. B. auf einer anderen Höhe auftaucht (Ionescu et al. 2014, 1326). Für die Optimierung neuronaler Netzwerke bedeutet das, aus 3D-Posen 2D-Werte $\mathrm{zu}$ extrahieren und $\mathrm{zu}$ abstrahieren, um ein prädiktives Modell menschlicher Körperposen herleiten zu können, dass auch in der offenen Umwelt (also in the wild) antizipationsfähig bleibt. ${ }^{14}$

14 Dies impliziert häufig das Auflegen einer „bounding box“ über mehrere Bilder sukzessiver Körperposen hinweg, die den Körper zentriert. Die sukzessiven Bilder werden durch künstliche neuronale Netzwerke verkettet, so dass ein statistischer Volumenwert für die diversen Posen entsteht. Dieser Wert wird durch Regressionsanalysen wieder als 2D-Grafik visualisierbar (Tekin et al. 2016; zur „bounding box“ sh. auch Engemann 2019). 
Die beiden Beispiele einer virtuellen Beobachtung zeigen, dass eine banale Grundunterscheidung wie Mensch/nicht-Mensch oder allgemeiner vertraut/unvertraut hier auf eine Komplexität von Bewegungsdaten und -auswertungen trifft, die allerdings selbst als Fremdreferenz in ihre Operation integrierbar sein müssen. Dies erstreckt sich nicht allein in der Komplexität eines hochtechnisierten Verfahrens. Denn die Banalität muss immer rückgebunden werden an eine Erwartungshaltung, ja an eine Integrationserwartung darüber, wie der Mensch selbst als Anderer in der Interaktion erkannt werden möchte. Gerade weil auch diese Form der Interaktion sich nicht der doppelten Kontingenz entledigen kann, stellen Medien der Virtualität das Paradoxon einer Komplexität des Banalen aus. Sie sind Medien für die werdende Fremdreferenz „Mensch“ in den Rekursionen des robotischen Systems. Diese Form des anwesenden NichtIch im Ich lässt sich mit Niklas Luhmann als „Interpenetration“ (1987, 290) bezeichnen. Anhand dieses Begriffes soll abschließend der Schritt von der Simulation banaler Bewegungen hin zum Embodiment in der Robotik gemacht werden.

\section{Banalität und Embodiment in der Robotik - ein Ausblick auf die Geste}

Die Interaktion menschlicher und maschineller Agenten hat zwar nicht dasselbe Kontingenzpotential wie die Interaktion zweier Menschen. Allerdings gilt es hier nicht, soziale Interaktionen unter Menschen als Maßstab für Mensch-Roboter-Interaktionen zu betrachten. Stattdessen wurde angenommen, dass Banalität zu einem entscheidenden Operationsmodus für die Ingenieurinnen der Robotik wurde, ja, dass die Ingenieur*innen selbst einen Blick auf das Banale richten müssen, um zu wissen, wie der Mensch als Umwelt des Roboters modelliert werden soll. Genau darin ist Banalität der Modus für eine Kontrolle des dauerhaften „lack of control“. Die Beispiele der virtuellen Beobachtung machten dabei deutlich, dass das, was unter Individuen als Projektion oder Intersubjektivität benannt wird, hier vom systematischen Analysieren und Synthetisieren von Bewegungen ausgeht, aus denen die Unterscheidungen vertraut/unvertraut oder bekannt/unbekannt hervorgehen.

Die Fremdreferenz „Mensch“ basiert dabei gänzlich auf (Sensor-)Daten, die nicht nur ein Konzentrat gespeicherter, digitalisierter Bewegungen darstellen. Sie hätten als Exempel des „technologisch Unbewussten“ auch nie Gegenstand des Bewusstseins werden können (Frizot 2003, 467), dienen aber nun dazu, ge- 
rade die Komplexität des Anderen (des Menschen als Umwelt) zu reduzieren (vgl. Kasprowicz 2019b). Wenngleich wir so etwas für psychische Systeme nicht annehmen können, weil diese in der Bewusstseinszeit operieren und nicht in der Simulationszeit des technischen Systems, so ist auch für sie eine Reduktion der Eigenkomplexität in der Interaktion notwendig. Erst unter diesen Bedingungen trete eine neue „Spezifikation der Körperbewegung“ ein, die eine „Interpenetration“, eine Anwesenheit im anderen System als Abwesendes ermöglicht (Luhmann 1987, 333). Dreh- und Angelpunkt dieser Grundlage von Interaktion ist nach Luhmann nicht die Intelligenz, auch nicht die Sprache, sondern der Körper:

Körper fordern wechselseitig ihre Reduktionsmöglichkeiten heraus. Sie können dies, wenn sie je eigene Komplexität präsentieren, vor allem im Sinne räumlicher Bewegungsmöglichkeiten, und damit Konditionierbarkeit ihrer selbstgeleisteten Reduktion in Aussicht stellen.

(Luhmann 1987, 333)

Mit anderen Worten: Die omnipräsente doppelte Kontingenz funktioniert nur durch das Anbieten von vereinfachten Bewegungsabläufen, von Banalitäten, deren kommunikative Notwendigkeit in der Inklusion des jeweils Anderen als anwesendem Abwesenden, als Differenz von Fremd- und Selbstreferenz liegt, die eine Adressierung des handelnden Körpers erst ermöglicht. Luhmann benennt für diesen körperlichen Vorgang selbst ein Medium, die Geste. Die Geste repräsentiere ein Medium, weil durch sie die innere moralische und emotionale Verfassung noch nah am körperlichen Ausdruck hänge und damit beobachtbar bleibe (Luhmann 1987, 334). ${ }^{15}$

Diese Kopplung von Geste und Ausdruck ist mit Blick auf die Robotik allerdings mehr als eine historische Analogie. Wie gezeigt wurde, erstreckt sich die Banalität nicht als Gemeinplatz weltlicher Erfahrungen. Stattdessen offenbart die Banalität die Komplexität der Kommunikation selbst, in dem sie eine körperliche Selbstreduktion zum Einspielen von Erwartungshaltungen voraussetzt, auf denen wieder neue Komplexitäten entstehen können - und damit auch eine neue Banalität (zu einer Gegenkonzeption, die das Missverhalten als notwendige Kategorie der proaktiven Technik fasst, vgl. Samuel 2014 et al.). Die

15 Bei Luhmann nimmt die Geste einen besonderen Raum ein, indem sie sich in Formen wie moralisch/unmoralisch, beherrscht/unbeherrscht codieren lässt. Interessanterweise änderte sich dies ab dem achtzehnten Jahrhundert, also jenem Jahrhundert der Massenalphabetisierung und der Psychologisierung des Romans. Es wäre nun die Differenz bewusst/unbewusst, die im Zuge der Introspektion und im Medium des Wortes dominiere und der die „Körpersprache“ kein „leistungsfähiges Äquivalent“ mehr bieten könne (Luhmann 1987, 335). 
Orte solcher gestenbasierten Banalitätsproduktionen kursieren durch Firmenpläne und Forschungszentren - sie stehen allerdings noch allzu oft in einer Forschungspolitik, die primär der Codierung innovativ/nicht-innovativ folgt. Was übergreifend von industriellen bis zu sozialen Robotern zu beobachten ist, was sowohl die Handreichung bei Greifarmrobotern wie das Anfahren bei Kinderrobotern umtreibt, ist das Erarbeiten von Kommunikation im Modus des Banalen.

An die Stelle eines progressiven und allzu anthropozentrischen Intelligenzbegriffes setzt die Banalität ein integratives Konstruktionsprinzip. Sicherlich sind Roboter nicht in dem Sinne selbstreferentiell wie es psychische Systeme sind, aber im Medium der Geste setzt sich immer die Welt des Handelnden in jene des Beobachtenden - oder anders: es wird eigenreduzierte Komplexität zugunsten einer Sinnvermittlung aufgedrängt. ${ }^{16}$ Das dies stets - gerade in der Multiplikation von Systemen - zu Problemen führen kann, ist sowohl von technischer wie von sozio-kultureller Seite unbestritten. Aber die zu Beginn besagte Funktion von Organisationen, solche Räume zu gewähren und gleichzeitig zu beschränken, kann ebenso als neuer Spielraum des Gestischen verstanden werden. ${ }^{17}$

Damit wäre dem Medium der Geste, ebenso wie der Körperpose oder der Bewegung, eine Bedeutung zugeordnet, die über ihre Kommunikationsfunktion hinausginge. Sie wäre ein Medium, an dem sich die Dualität von maschinellen und menschlichen Agenten zugunsten eines neuen Embodiment-Begriffes destabilisiert. Die Bewegungsmodi und Gesten als Präsentationsmöglichkeiten des Körpers finden in der virtuellen Beobachtung einen Ort, an dem Kommunikation als Kondensat einer neuen Banalität entsteht. An der Stelle einer posthumanistischen Überhöhung erfolgt das Einspielen von Banalitäten, die eine Mittel-Zweck oder Master-Slave-Beziehung in der Robotik nicht ganz hinter sich lassen, aber doch zeitweise suspendieren. Oder anders: Um Banalitäten zu operationalisieren, müssen Robotik-Ingenieur`innen lernen, auf die Sachen selbst zurückzugehen.

16 Die Mitteilungen zur Aktualisierung der Sicherheitsmaßnahmen in der industriellen Robotik sind hierzu ein eindrückliches Beispiel, sh. dazu DGUV 2017.

17 Dies lässt sich an Beispielen wie dem Tragen einer Tischplatte mit semiautonomen, bipedalen Robotern und menschlichen Kollaborateuren aufweisen, wenn während der Bewegung die Zuweisungen des Führenden und des Geführten wechseln, sh. dazu mit einem Schwerpunkt auf dem Modellierungsaspekt sowie auf eine Episteme der Intuition (Kasprowicz 2018 und 2020). 


\section{Literatur}

Alač, Morana. „Social robots: Things or agents?”. Al \& Society 31.4 (2016): 519-535.

Baecker, Dirk. Form und Formen der Kommunikation. Frankfurt a.M.: Suhrkamp, 2005.

Bannon, Liam. „Reimagining $\mathrm{HCl}$ : Toward a More Human-Centered Perspective”. Interactions 7.8. (2011): 5-57.

Bergmann, Werner, und Gisbert Hoffmann. „Selbstreferenz und Zeit: Die dynamische Stabilität des Bewusstseins“. Husserl Studies 6 (1989): 155-175.

Blumenberg, Hans. „Vorbemerkungen zum Wirklichkeitsbegriff“. Akademie der Wissenschaften und der Literatur. Abhandlungen in der Geistes- und Sozialwissenschaftlichen Klassen 4 (1974): 3-10.

Bortot, Dino et al. „Effizienzsteigerung durch Bewegungsanalyse und -modellierung der Mensch-Roboter-Kooperation“. Zeitschrift für Arbeitswissenschaft 60.2 (2010): 65-75.

Bubb, Heiner, und Florian Fritzsche. „A Scienfitic Perspective of Digital Human Models: Past, Present, and Future“. Handbook of Digital Human Modelling. Hg. Vincent Duffy. Boca Raton; FL: CRC Press, 2009. Kap. 3-1: 3-29.

Cañameo, Lola, und Matthew Lewis. „Making New ,New Al‘ Friends: Designing a Social Robot for Diabetic Children from an Embodied AI Perspective“. International Journal for Social Robotics 8 (2016): 523-537.

Chaumette, François, Seth Hutchinson und Peter Corke. „Visual Servoing“. Springer Handbook of Robotics. 2. Aufl. Hg. Bruno Siciliano und Oussama Khatib. Heidelberg: Springer, 2016. 841-866.

Christaller, Thomas et al. (Hg.). Robotik. Perspektiven für menschliches Handeln in der zukünftigen Gesellschaft. Wiesbaden: Springer, 2001.

Decker, Michael. „Service-Roboter im Blick der Technikfolgeabschätzung“. Technikfolgeabschätzung - Theorie und Praxis 20.2 (2011): 76-79.

DGUV (Deutsche Gesellschaft für Unfallversicherung). „Kollaborierende Roboter. Planung von Anlagen mit der Funktion ,Leistungs- und Kraftbegrenzung““, 2017 https://www.dguv.de/ medien/fb-holzundmetall/publikationen-dokumente/infoblaetter/infobl_deutsch/080_ roboter.pdf (4. November 2018).

Engemann, Christoph. „Rekursionen über Körper. Machine-Learning Trainingsdatensätze als Arbeit am Index". Machine Learning. Medien, Infrastrukturen und Technologien der Künstlichen Intelligenz. Hg. Christoph Engemann und Andreas Sudmann. Bielefeld: transcript, 2019. 247-268.

Ernst, Christoph et al. „Künstliche Intelligenzen. Einleitung in den Schwerpunkt“. Zeitschrift für Medienwissenschaften 21.2 (2019): 10-19.

Esposito, Elena. „Artificial Communication? The Production of Contingency by Algorithms“. Zeitschrift für Soziologie 46.4 (2017): 249-265.

Føllesdal, Dagfinn. „Husserl’s Notion of Noema“. Journal of Philosophy 66.20 (1969): 680-687.

Frizot, Michel. „Der menschliche Gang und der kinematographische Algorithmus“. Diskurse der Fotografie. Fotokritik am Ende des fotografischen Zeitalters. Hg. Herta Wolf. Frankfurt a.M.: Suhrkamp, 2003. 456-478.

Gammieri, Luigiet al. „Coupling of a Redundant Manipulator with a Virtual Reality Environment to Enhance Human-robot Cooperation“. Procedia CIRP 62 (2017): 618-623.

Genz, Julia. Diskurse der Wertung. Banalität, Trivialität und Kitsch. München: Wilhelm Fink, 2011. 
Gratch, Jonathan et al. „Exploring the Implications of Virtual Humans Research for HumanRobot Teams“. Virtual, Augmented and Mixed Reality. $7^{\text {th }}$ International Conference, VAMR. Los Angeles 2.-7.8.2015. Hg. Randall Shumaker und Stephanie Lackey. New York u. a.: Springer, 2015. 186-196.

Haddadin, Samin, Alin Albu-Schäffer und Gerd Hirzinger. „Requirements for Safe Robots: Measurements, Analysis and New Insights“. The International Journal of Robotics Research 28.11-12 (2009): 1507-1527.

Haddadin, Sami et al. „It Is (Almost) All about Human Safety: A Novel Paradigm for Robot Design, Control and Planning“. Computer safety, reliability and security: 32nd International Conference. Hg. Friedemann Bitsch, Jérémie Guiochet und Mohamed Kaâniche. Berlin, Heidelberg: Springer, 2013. 202-215.

Hasse, Jürgen. Die Aura des Einfachen. Mikrologien räumlichen Erlebens. Freiburg: Verlag Karl Alber, 2017.

Husserl, Edmund. Die Idee der reinen Phänomenologie. Fünf Vorlesungen. Husserliana Bd. II. Hamburg: Meiner, 1973 [1907].

Husserl, Edmund. Logische Untersuchungen. 2. Band. 1. Teil. Hg. Ursula Panzer nach der Ausgabe Husserliana Bd. XIX 1. Hamburg: Meiner, 2009 [1901].

Ionescu, Catalin et al.: „Human 3.6M: Large Scale Datasets and Predictive Methods for 3D Human Sensing in Natural Environments“. IEEE Transactions on Pattern Analysis and Machine Intelligence 36.7 (2014): 1325-1339.

Kanderske, Max, und Tristan Thielmann. „Simultaneous localization and mapping and the situativeness of a new generation of geomedia technologies“. Communication and the Public 4.2 (2019): 118-132.

Kasprowicz, Dawid. „Das Interface der Selbstverborgenheit. Szenarien des Intuitiven in Mensch-Roboter-Kollaborationen“. Unterwachen und Schlafen. Anthropophile Medien nach dem Interface. Hg. Michael Andreas, Dawid Kasprowicz und Stefan Rieger. Lüneburg: Meson, 2018. 159-188.

Kasprowicz, Dawid. Der Körper auf Tauchstation. Zu einer Wissensgeschichte der Immersion. Baden-Baden: Nomos, 2019a.

Kasprowicz, Dawid. „Von alten Besen im System und neuen Körpern im Loop. Bildgebungsverfahren in der Mensch-Roboter-Kollaboration“. Augenblick. Konstanzer Hefte zur Medienwissenschaft 73 (2019b): 43-58.

Kasprowicz, Dawid. „Encoding Proximity - Intuition in Human-Robot-Collaborations“. Affective Media and Policy. Hg. Bernd Bösel und Serjoscha Wiemer. Lüneburg: Meson Press, 2020, im Print.

Kasprowicz, Dawid. „Soma in Space. Leibkonstruktionen in ,Abwesenheit“ der Technik“. Aller guten Dinge sind frei? Unbehagen und Utopie in der technisierten Welt. Hg. Dennis Niewerth und Stefan Rieger. Münster: Lit Verlag, in Vorb.

Luhmann, Niklas. Soziale Systeme. Grundriß einer allgemeinen Theorie. Frankfurt a.M.: Suhrkamp, 1987.

Luhmann, Niklas. Die Gesellschaft der Gesellschaft I. Frankfurt a.M.: Suhrkamp, 1997a. Luhmann, Niklas. Die neuzeitliche Wissenschaft und die Phänomenologie. Wien: Picus, 1997b. Luhmann, Niklas. Einführung in die Systemtheorie. Heidelberg: Carl Auer Verlag, 2002. Mackenzie, Adrian. Machine Learners. Archaeology of Data Practice. Cambridge, MA: MIT Press, 2017. 
Meister, Martin. „Mensch-Technik-Interaktivität mit Servicerobotern. Ansatzpunkte für eine techniksoziologisch informierte TA der Robotik“. Technikfolgeabschätzung - Theorie und Praxis 20.1 (2011): 46-52.

Miller, Tim. „Explanation in Artificial Intelligence: Insights from the Social Sciences“. Artificial Intelligence 267.2 (2019): 1-38.

o. A. „Banal“. Pierer's Universal-Lexikon, Band 2. Altenburg, 1857. 264. http://www.zeno.org/ nid/20009463895 (23. Januar 2020).

Onnasch, Linda, Xenia Maier und Thomas Jürgensohn: „Mensch-Roboter-Interaktion: Eine Taxonomie für alle Anwendungsfälle“. Hg. Bundesanstalt für Arbeitsschutz und Arbeitsmedizin (BAUA). Dortmund, 2016. https://www.baua.de/DE/Angebote/Publikatio nen/Fokus/Mensch-Roboter-Interaktion.pdf?__blob=publicationFile\&v=6 (6. November 2018).

Oudshoorn, Nelly, und Trevor Pinch. „Introduction: How Users and Non-Users Matter“. How Users Matter. The Co-Construction of Users and Technology. Hg. Nelly Oudshoorn und Trevor Pinch. Cambridge, MA: MIT Press, 2005. 1-28.

Paauwe, Robert et al. „Designing Robot Embodiment for Social Interaction: Affordances Topple Realism and Aesthetics“. Internation Journal of Social Robotics 7 (2015): 697-708.

Samuel, Bianchini et al. „The Misbehavior of Animated Object“. 8th International Conference on Tangible, Embedded and Embodied Interaction (TEl'14), 16-19.02.2014, Munich. Hg. ACM. New York: ACM Press, 2014. 381-384.

Sárándi, Istvan et al. „How Robust Is 3D Human Pose Estimation to Occlusion?“. International Conference on Intelligent Robots and Systems (IROS'18.) Hg. IEEE. New York, 2018. https://arxiv.org/abs/1808.09316 (17. Dezember 2019).

Sartre, Jean-Paul. Das Imaginäre. Phänomenologische Psychologie der Einbildungskraft. Hamburg: Rowohlt, 1971 [1940].

Schirra, Frank. Entwicklung eines Systems zur immersiven Arbeitssystemgestaltung mit CAD-Schnittstellen. Dissertation Universität Stuttgart, 2009.

Sprenger, Florian. Epistemologien des Umgebens. Zur Geschichte, Ökologie und Biopolitik künstlicher Environments. Bielefeld: transcript, 2019.

Stilgoe, Jack. „Machine learning, social learning and the governance of self-driving cars“. Social Studies of Science 48.1 (2018): 25-56.

Tekin, Bugra et al. „Direct Prediction of 3D Poses from Motion Compensated Sequences“. 2016 IEEE Conference on Computer Vision and Pattern Recognition (CVPR), 27.-30.06. 2016, Las Vegas Hg. IEEE. New York, 2016. 991-1000.

Thiele, Thomas, Tobias Meisen und Sabina Jeschke. „Smart Factory“. Handbuch Virtualität. Hg. Dawid Kasprowicz und Stefan Rieger. Wiesbaden: Springer, 2020. 79-96.

Thomasset, Vincent, Stéphane Caron und Vincent Weistroffer. „Lower body control of a semiautonomous avatar in Virtual Reality: Balance and Locomotion of a 3D Bipedal Model“. Proceedings of the 25th ACM Symposium on Virtual Reality Software and Technology, VRST, 12.-15.11., Parramatta, NSW, Australia. Hg. ACM. New York: ACM Press, 2019. 1-11. doi: $10.1145 / 3359996.3364240$.

Tykal, Martin, Alberto Montebelli und Ville Kyrki. „Incrementally Assisted Kinaesthetic Teaching for Programming“. $11^{\text {th }}$ ACM/IEEE International Conference on Human-RobotInteraction, 07.-10.03.2016, Christchurch, New Zealand. Hg. ACM/IEEE. doi: 10.1109/ HRI.2016.7451753.

von Foerster, Heinz. „Erkenntnistheorien und Selbstorganisation“. Der Diskurs des radikalen Konstruktivismus. Hg. Siegfried J. Schmidt. Frankfurt a.M.: Suhrkamp, 1987. 133-158. 
von Foerster, Heinz. „Abbau und Aufbau“. Lebende Systeme. Wirklichkeitskonstruktionen in der systemischen Therapie. Hg. Fritz B. Simon. Frankfurt a.M.: Suhrkamp, 1997. 33-51. Weber, Jutta, und Fabian Kröger. „Autonomous Driving and the Transformation of Car Cultures“. Transfers 8.1. (2014): 1-23.

Zerilli, Tom et al. „Transparency in Algorithmic and Human Decision-Making: Is There a Double Standard?“. Philosophy \& Technology 32 (2019): 661-683. 
